\title{
FOCUS ON ECOLOGICAL IMPROVEMENTS IN THE DESIGN OF A BEACH STABILIZATION PROJECT
}

\author{
Kevin Maclntosh, Baird \& Associates, Barbados, kmacintosh@baird.com \\ Dr. Tundi Agardy, Baird \& Associates, USA, tagardy@baird.com \\ Seth Logan, Baird \& Associates, Canada, slogan@baird.com
}

\begin{abstract}
SHORT SUMMARY
Baird harnessed the latest technology to improve the beach while minimizing environmental impacts and improving habitat wherever possible at a west coast location in Barbados. Baird used an Echoscope to precisely map bathymetry, living reef, and voids in relic reef. Following numerical and physical modeling, underwater structures for beach stabilization were specifically designed to accommodate coral transplants and lab grown corals. This first phase of shoreline stabilization creates new opportunities for enhancement, training, and education. Subsequent monitoring of biodiversity will measure the rate of reef recovery. Turbidity monitoring, as well as rainfall and surface run-off rates, will provide much needed information regarding the relative impacts of wave sediment resuspension and surface run-off on coral health.
\end{abstract}

\section{SYNOPSIS}

Over the past 36 years, Baird has been involved in the planning, design and construction of numerous beach stabilization projects around the world, with the scope/scale of these projects ranging from individual properties $(<100$ $\mathrm{m}$ ) to extended shoreline reaches (up to $7 \mathrm{~km}$ ). These projects have been implemented to address a wide range in objectives, including beach stabilization, erosion and flood protection, improved recreational amenities, and improved swimmer safety, as well as habitat protection, environmental restoration, and ecological enhancement. Utilizing the latest technology and modeling capabilities, the shoreline stabilization projects on Caribbean islands have led to positive outcomes, including improved water quality, enhanced coral reef health, biodiversity conservation, and increased recreational and aesthetic value for visitors and residents.

Of particular interest is our application of a range of complementary methodologies and technologies to support the design, construction and monitoring of such projects, including: high resolution site surveys using an unmanned aerial vehicle (UAV) and multi-beam sonar (MBS) systems (used to map the precise location of existing coral and habitat); detailed field assessments of nearshore benthic and marine habitats and site-specific ground-truthing; and numerical and physical modeling of littoral processes. While the role of numerical modeling in the design of these projects has increased significantly over the past 15 years, new field data collection techniques, physical modeling and practical experience continue to provide invaluable input to the design process for beach stabilization and coastal enhancement projects.

This paper presents a project currently underway in Barbados where concerns for maintaining and improving the marine environment have driven the entire design process in a resort redevelopment. This enhancement-oriented design process has included: determining the optimal position, size and design of the coastal structures; inclusion of underwater structures specifically for habitat enhancement that will increase fish productivity; a 3 year monitoring program that will document the level of success of each structure in meeting shoreline stabilization and environmental enhancement aims; a developer-funded research agenda that allows training of local marine biologists and engineers; and an educational / awareness-raising component for hotel guests once the facility is operational.

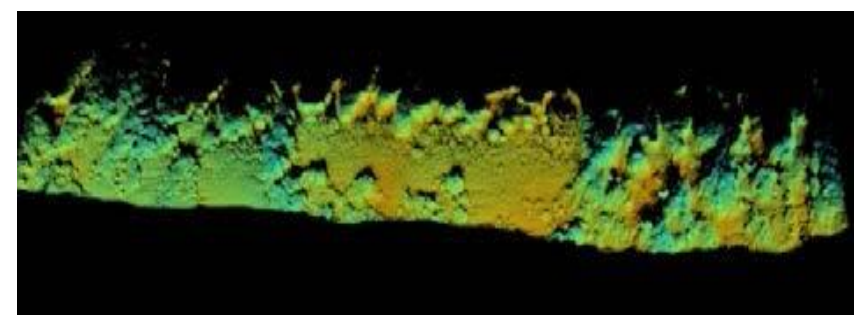

Figure 1. Echoscope 3D imagery of nearshore site showing coral formations

We utilized a cutting-edge Echoscope to precisely map the location of each living coral colony of a certain size, as well as to roughly quantify the extent and location of voids in the relic reef that act as important fish nursery habitat. The findings of the extensive field assessments and physical model essentially led to a complete redesign of the original concept, with the repositioning of marine structures focused on minimizing habitat impacts and creating new opportunities for environmental enhancement, training, and education. Optimally positioned submerged breakwaters stabilize the beach, keeping sediment off boulder corals that are already stressed by the turbidity in Barbados waters during certain times of the year. Additional small underwater structures increase habitat for fishes and other marine life, and allow for viewing opportunities for Barbados residents and hotel guests.

Mapping with the Echoscope allowed determination of exact location of corals at the site, providing important baseline information to assess future performance of structures and overall condition of the nearshore reef. It also provided data for the physical model that supported the project redesign and optimization. Finally, the Echoscope mapping allowed precise quantification of the number and types of corals to be moved in translocation. 


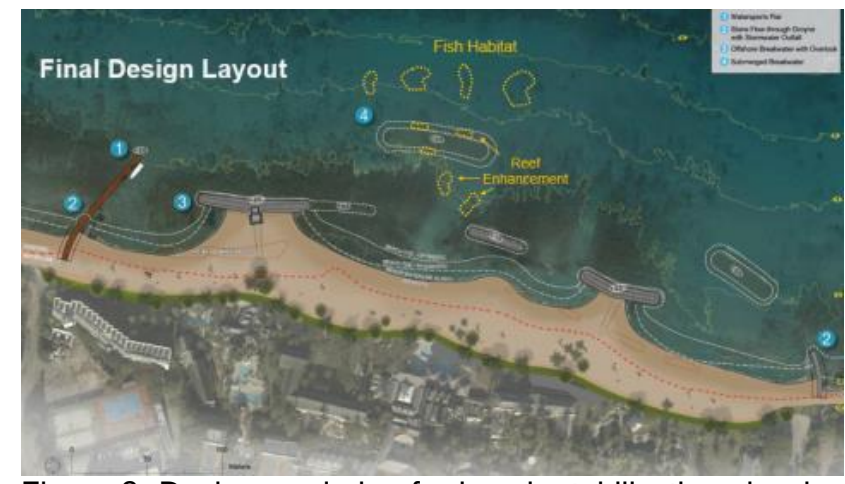

Figure 2. Design rendering for beach stabilization showing submerged breakwaters and enhancement structures.

One of several unique features of the enhancement project is the transplanting of hard and soft corals, taken from the construction footprint, onto plates built into the breakwater at various water depths and wave exposure positions. These will then be monitored for several years in terms of performance (coral survivorship, growth, and resilience), to further inform the community of Caribbean coral restoration experts on the optimal siting of translocated corals. In addition to corals from the existing location, the intent is to transplant coral fragments grown in a lab to the breakwater. This work is supported by the private sector and is being completed by a combination of private and public sector scientists and engineers as well as graduate students from the local university. The intent is to provide an excellent educational training opportunity as well as necessary research into the impacts of storm water and wavegenerated turbidity on coral health.

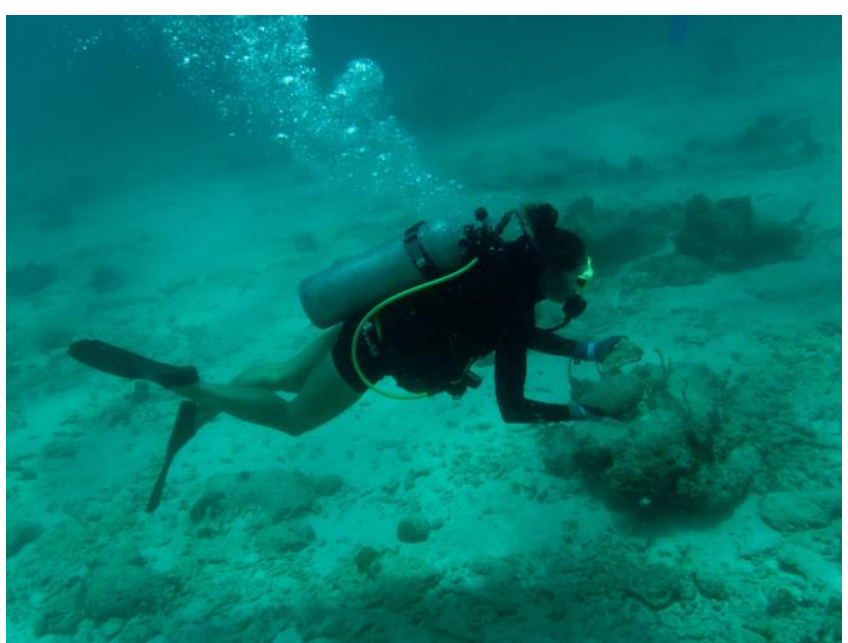

Figure 3. Corals being moved to transient recipient site until being relocated to the specially-designed submerged breakwater.
In addition to the coral survivorship and associated biodiversity / fish monitoring, the research agenda includes an in-depth study of biophysical conditions that contribute to coral reef and coastal ecosystem health, productivity, and resilience. The three-year fully funded research protocol includes ongoing monitoring of waves, currents and turbidity in addition to regular monitoring of fish, sponges, algae, and coral abundance and condition. In particular, the research program aims to resolve issues relating to water quality and its effect on nearshore reef ecosystems. Public entities are often worried about run-off and its effect on coastal water quality and on coral reef health, but very few data exist on sources of nutrients and sediments. This research will allow us to differentiate turbidity that originates from swell-induced resuspension of bottom sediments versus run-off from surface water. This research agenda is intended to document performance of enhancement measures and build the capacity of local institutions to guide future development and redevelopment in Barbados, thereby providing guidance for similar efforts throughout the Caribbean. 